\title{
Visualization of evaporative convection in minute drops by laser shadowgraphy
}

\author{
N. Zhang ${ }^{a}$ and Wen-Jei Yang \\ Department of Mechanical Engineering and Applied Mechanics, University of Michigan, Ann Arbor, Michigan 48109 \\ (Received 4 June 1982; accepted for publication 28 September 1982)
}

A very simple optical procedure is developed to investigate the morphology of natural convection and the dynamic behavior of drops evaporating on a plate by means of a laser shadowgraphic system. This method is the only nonintrusive means of visualizing flow patterns in a time-varying "lens" shaped system. Its uniqueness and superiority are discussed.

PACS numbers: $68.10 . \mathrm{Jy}$

\section{INTRODUCTION}

Flow phenomena on a microscopic scale, which can not be viewed with the naked eye, take place on the surface of liquid layers or liquid drops undergoing evaporation. Their occurrence is attributed both to the surface tension mechanism due to local variations in the surface temperature and to the buoyancy mechanism resulting from evaporative cooling.

Spangenberg and Rowland' confirmed, photographically, the presence of a tesselated network on the surface of water in deep tanks by means of an ordinary Schlieren technique. This method has thus emerged as a convenient and valuable experimental tool for investigating the morphology of evaporative convection. Berg $e t$ al. ${ }^{2}$ used the optical method to reveal a variety of flow patterns in an evaporating liquid layer. The work was undertaken to obtain qualitative information concerning both the structure and the scale of these flows, and particularly the manner in which they are affected by fluid properties, fluid depth, and the presence of surface contamination.

A minute drop placed on a flat plate takes the form of a spherical segment (at least very close in most cases) due to the action of surface tension. Since it is like a thin lens with a convex surface, parallel incident rays intersect at a single point (or at least in a very small region) after transversing the drop. This point is known as the principal focal point of the lens. When the drop reduces in size with evaporation, the focal point moves. The optical methods are the only nonintrusive means of visualizing flow phenomena in a drop evaporating on a flat plate. However, both the Schlieren method and the MachZehnder interferometry are unsuitable for this purpose because both techniques can not be applied to an object with an unsteady focal point. The only optical method available is shadowgraphy which is nonsensitive to a change in the focal point of the object.

Shadowgraphy is known to be the simplest of all optical visualizing methods. It requires only a collimating lens to make the light parallel. When passing through the field under investigation, the individual light rays may be refracted or diffracted and deviate from their original path. A darker region on the screen represents the shadow of the disturbance through which a deflected ray has passed.
The shadow method is roughly sensitive to changes in the second derivative of density of the field. ${ }^{3}$ When the density increases, the disturbance acts like a convex lens. On the other hand, in the case of a decrease in density, the disturbance exerts the effect of a concave lens. In the presence of turbulent motions in the field, the fluctuating density variation acts as a distribution of tiny convex and concave lenses which are continuously changing in shape and location. The effect will be utilized here to investigate the interfacial turbulence and internal convection in drops evaporating on a plate.

\section{APPARATUS}

Laser shadowgraphy was achieved using an apparatus consisting of a laser light, a test plate and two aluminized mirrors, a screen, and a camera, as illustrated in Fig. 1. A minute drop was placed on an optically flat glass plate of $102 \times 127 \mathrm{~mm}$ and $1.5 \mathrm{~mm}$ thickness for evaporation. A C. W. Radiation Model SP 2, 2.5-mW cylindrical helium-neon laser was used as the light source. The two mirrors were aluminized on the surface nearest to the objects that were viewed so that light could be reflected with no refraction. They were mounted in parallel on a specially designed frame set at $45 \mathrm{deg}$. The test plate was inserted into the vertical light beam between the two mirrors. The mirror arrangement was used to obtain a horizontal view of the evaporating drop. The optical path from the lower mirror was horizontal and intercepted by a vertical screen. The image on the screen was recorded by a $16-\mathrm{mm}$ Bolex H16 EBM movie camera having a Nikon Nikkor 300-mm $1: 4.5$ lens. A Kalt 72-mm CloseUp Dioptor lens was mounted on top of the Nikon lens to photograph the air-liquid and liquid-glass interfaces of an evaporating drop. A drop was carefully placed on the test plate by means of a $50-\mu 1$ Monoject microsyringe. The needle tip was allowed to touch the plate surface before and during the injection to permit the formation of a calm, unsplashing drop.

\section{RESULTS AND DISCUSSION}

Consider the volume of an evaporating drop to be placed in the collimated light beam of a shadowgraphic 


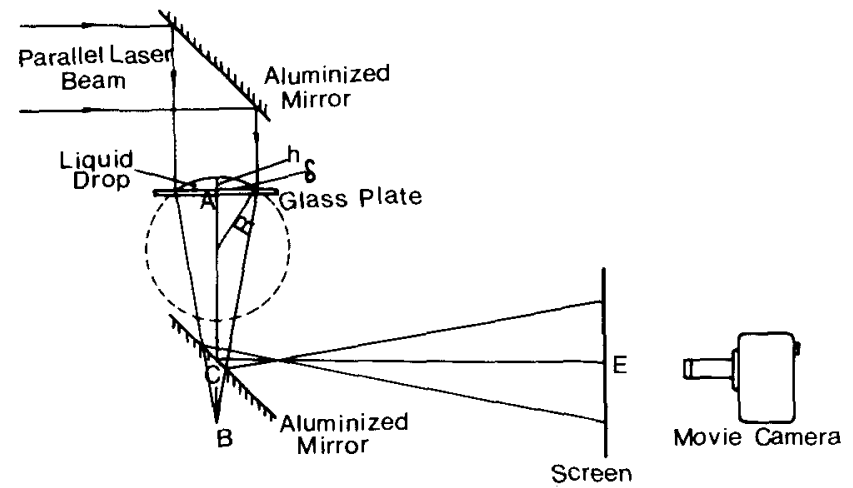

FIG. 1. A schematic of the laser shadowgraphic system.

system so that the light traverses the volume from top to bottom as shown in Fig. 1. The image brightness at a given point is linearly related to the integral average of the second derivative of the refractive index over the thickness of the drop along the passage of a ray. Thus, a field of uniform refractive-index gradient, i.e., zero second derivative, yields an image of uniform (mean) brightness. The entire image, being a map of the distribution of the (depth average) second derivative of the refractive index, represents also the (depth average) second derivative of the temperature in the evaporating drop, since the refractive index is a function of the temperature.

The photographs by laser shadowgraphy exhibited two sets of edges (i.e., peripheries): one appearing inside the drop image and the other outside the drop image, as schematically illustrated in Fig. 2. The inner periphery is formed by a shadow, which is cast when light hits the edge of the test object (in this case, a drop). The rings within the inner periphery are caused by the Fresnel diffraction of circular aperture. ${ }^{4}$ This set of rings propagated outward as the drop evaporation proceeded, i.e., as the drop periphery moved inward. Therefore, the speed of the moving rings reflected the contracting velocity of the drop periphery. The outer periphery is caused by the refraction of light when the light passes two media with different densities, the two media being air and liquid in this experiment. The light is refracted by the change in density between the air and liquid at the air-liquid interface. Therefore, the unevenness of the interface is reflected by the shape of the outer periphery. The rings close to the outer periphery are also induced by the Fresnel diffraction.

Figures 3(a) and (b) illustrate instant laser shadowgraphs of cyclohexane and acetone drops, respectively, which evaporated on an unheated horizontal plate (with uniform temperature). The dark circle at the center of each photo represented the image of each drop. The rectangle outside the circle was a piece of transparent Scotch tape attached to the center of the screen. This was done to balance the illumination of the other portion of the screen and to enhance the contrast of the inner rings which were printed separately in Fig. 3(c). The inner rings in Fig. 3(c) were similar in appearance for all types of evaporation since the inner rings showed the drop pe-

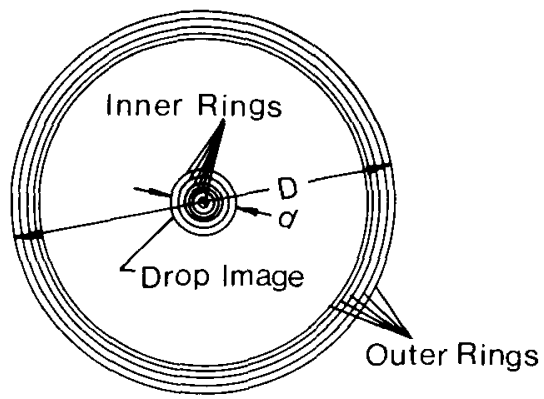

FIG. 2. Two sets of rings in a drop image on the screen

riphery which remained practically circular in shape during the evaporation of tiny minute drops.

For an unstable-type evaporation, the drop surface is rippled, resulting in a jagged periphery as in Fig. 3(b) for acetone. On the other hand, a stable-type evaporation is characterized by a very smooth surface causing the outer periphery of the drop image to be in an almost circular shape as seen in Fig. 3(a) for cyclohexane. In between the two evaporation types, there exists a substable type evaporation whose interfacial patterns are in between the two extremes. The shape of the outer periphery is neither circular nor spiked. A group of liquids such as benzene, chloroform, ethyl acetate, and methyl chloride belongs to this category. There is no flow pattern visualized inside the outer periphery of the drop image since no appreciable temperature variation exists within the drops on an unheated plate.

Figure 4 is the shadowgraph of a carbon tetrachloride drop evaporating on an inclined flat plate. A strip of thermofoil heater was attached to the underside of the glass plate which was maintained at a temperature gradient of $1.57^{\circ} \mathrm{C} / \mathrm{mm}$. The plate temperature at the north pole of the drop was measured to be $51.3^{\circ} \mathrm{C}$. The south pole was the cold end. The plate temperature decreased from the north to south poles at a gradient of $1.57^{\circ} \mathrm{C} / \mathrm{mm}$. The
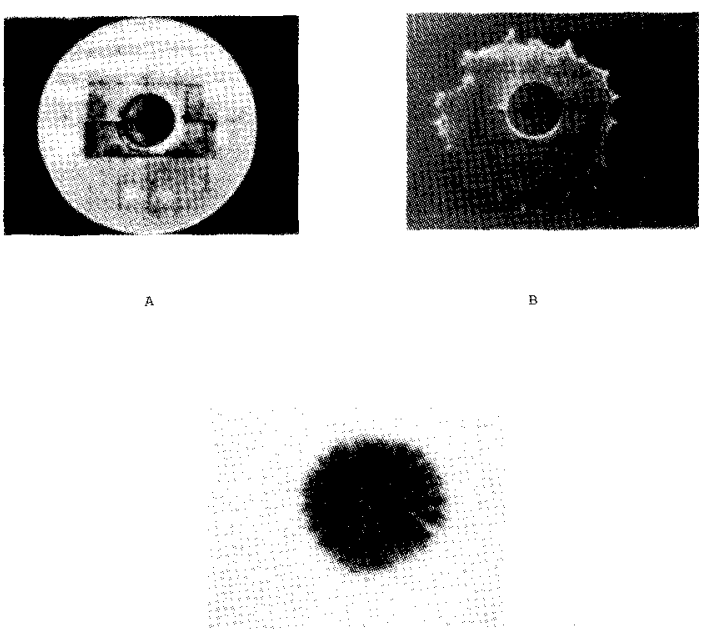

FiG. 3. Instant laser shadowgraphs of (a) cyclohexane, (b) acetone drops evaporating on a test plate, and (c) image of drop periphery on an unheated horizontal plate. 


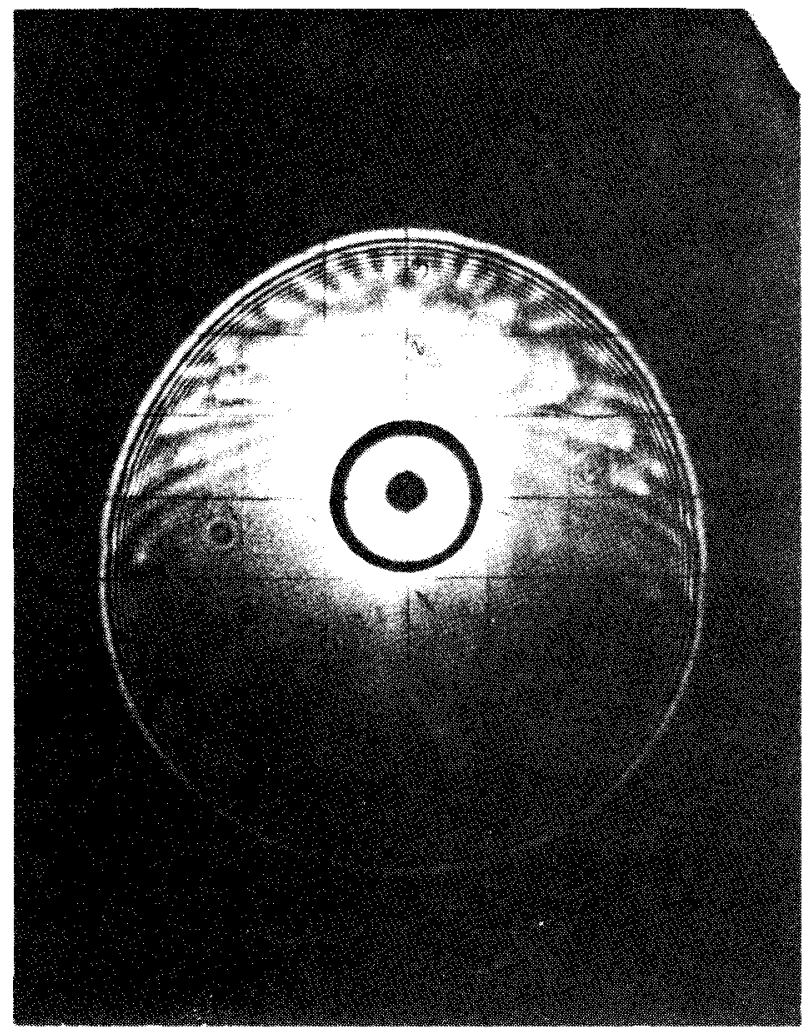

FIG. 4. Instant laser shadowgraph of a carbon tetrachloride drop evaporating on a test plate with an almost linear temperature field (temperature increasing downward).

plate was tilted $7 \mathrm{deg}$ from a horizontal position with the cold side up such that the drop could stay at rest on the plate surface during evaporation. The carbon tetrachloride took an egg shape due to the action of gravity, i.e., the small end on top (toward the cold side) and the large end at bottom (toward the warm side). Should the plate be held horizontal, the drop would not stay put but crawl toward the cold side of the plate due to the action of surface tension. The evaporation process is controlled by the surface tension-driven convection in the northern hemisphere (cold side) of the drop and by the buoyancydriven convection in the southern hemisphere (warm side). The warm side has a strong buoyancy-driven convection, causing a good mixing of the liquid. Therefore, the liquid temperature becomes rather uniform, resulting in a very small variation in the refractive index as indicated by the nearly uniform dark image in the shadowgraph. On the other hand, the surface tension-driven convection produces only poor liquid mixing in the cold side. As a result, a large temperature variation causes a large variation in the refractive index, thus producing a featherlike pattern in the shadowgraphic image. In order to ascertain that the outer periphery and its neighboring rings are a result of the interfacial pattern and not that of a variation in the refractive index, a needle with a very fine tip was used to very lightly poke a point on the drop surface. Poking anywhere on the drop surface of any type of evaporation would produce a substantial disturbance on the entire outer periphery and the rings. The observation suggests that the outer periphery is caused not by a refractive index change but by an interfacial turbulence. On the other hand, the feather-like pattern in the shadowgraphic image of Fig. 4 is generated by a large variation in the refractive index, since a poking of the drop surface causes a disturbance in only the outer periphery and its rings and not the feather-like pattern.

A method was developed to determine the drop volume-time history from the shadowgraph: First, the diameter of the liquid-solid interface on the test plate $\delta$ and that of the outermost ring on the screen $\mathrm{D}$ were measured. The distances $A C$ and $C E$ are known, where $A$ denotes the drop center on the test plate while $C$ and $E$ are its images on the plate front mirror and the screen, respectively, as shown by Fig. 1. Then, by a simple geometric relationship, the focal length of the $\operatorname{drop} f$, which is equal to $A B$, can be expressed as

$$
f=A B=\frac{\delta(A C+C E)}{D+\delta} .
$$

This equation also applies when $B$ falls between $A$ and $C$. Now, one can evaluate the radius of the spherical segment, $R$, using the relationship

$$
\frac{1}{f}=(n-1) \frac{1}{R},
$$

where $n$ is the refractive index. For the liquids tested here, $n$ varies from 1.3 to 1.5 . Then, the volume of the drop can be determined as

$$
V=\pi h^{2}\left(R-\frac{h}{3}\right),
$$

wherein the height of the spherical segment is

$$
h=R-\sqrt{R^{2}-(\delta / 2)^{2}} .
$$

A typical result of the volume-time history is presented in Fig. 5 wherein $V_{0}$ is the initial volume; $t$, time; and $\tau$, the drop lifetime. It shows that the volume-time history

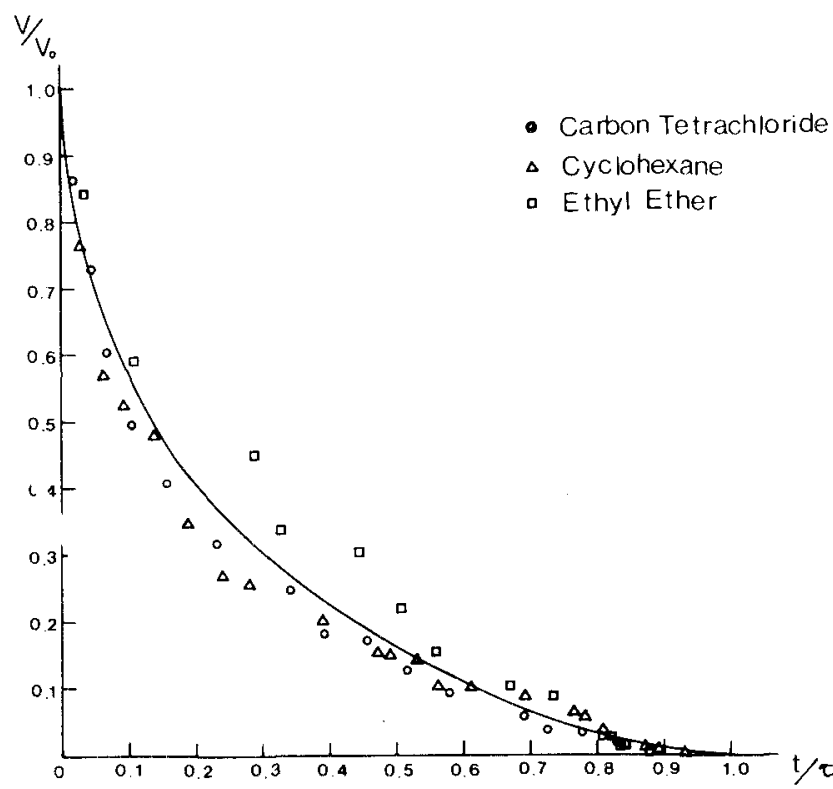

FiG. 5. Time history of drop size for stable-type evaporation. 
of the stable-type evaporation can be correlated by a monotonic curve (shown as a solid line). This is not the case in the unstable-type drop evaporation ${ }^{5}$ which exhibits three distinct stages. It is worth noting that the unsteadiness of the focal point can be realized from Eq. (1). As drop evaporation takes place, both $D$ and $\delta$ vary with time, resulting in a timewise variation of $f$, since $A C$ and $C E$ are both fixed.

\section{CONCLUSIONS}

The merits of shadowgraphy as applied to the evaporation of a drop on a flat plate include:

(i) Due to a change in the focal point of the drop (which behaves like a "lens") as evaporation proceeds, no optical methods other than shadowgraphy can be utilized for the visualization study of interfacial structure. The technique is not suited for the interfacial study of an evaporating liquid pool (with a flat interface).

(ii) The instantaneous drop size can be determined using a simple geometric relation between the drop and its image.

(iii) By a continuous recording of image change the dynamic behavior, such as the volume-time history, of a drop evaporation process may be analyzed.

(iv) During the evaporation process, changes in the half-chord $h$ and the sagitta $\delta$ of a drop (treated as a spherical segment) with time can be measured very accurately from the image recording. The use of an optical micrograph is not suitable for unsteady measurements. The location of the screen may be changed to enlarge or reduce the dimension of the drop image on the screen. Therefore, the accuracy in measurements of volume versus time surpasses that by direct photography commonly employed in the study of drop evaporation.

(v) The interfacial movement during drop evaporation is exhibited in the shape of the outer periphery of the image.

(vi) The convective pattern inside a drop evaporating on a heated plate may be visualized when a change in the refractive index is large in the presence of a large temperature variation.

\section{ACKNOWLEDGMENT}

This research work was supported by the National Science Foundation under Grant No. ENG-7816972.

\footnotetext{
a) Visiting scholar on leave from the Department of Thermal Engineering, Tsinghua University, Beijing, China.

W. B. Spangenberg and W. R. Rowland, Phys. Fluids 4, 743 (1961).

2 J. C. Berg, M. Boudart, and A. Acrivos, J. Fluid Mech. 24, 721 (1961).

'W. Merzkirch, Flow Visualization (Academic. New York. 1974). p. 80.

${ }^{4}$ B. Rossi, Optics (Addison-Wesley, Reading, Massachusetts, 1967). Chap. 4.

${ }^{5}$ N. Zhang and W. J. Yang, J. Heat Transfer 104 (to appear 1982).
} 